\title{
FUNCTIONAL COMPOSITES FOR ENERGY HARVESTING APPLICATIONS WITH LOW-FREQUENCY MAGNETIC ACTUATION
}

\author{
$\underline{\text { Michele Rosso }}{ }^{*}$, Raffaele Ardito, Alberto Corigliano \\ Department of Civil and Environmental Engineering - Politecnico di Milano, Piazza Leonardo da \\ Vinci 32, Milano, Italy \\ *michele.rosso@polimi.it
}

The increasing necessity of IoT, encouraged the research world to develop MEMS autonomous sensors in order to create a large network of smart communicating devices. To this purpose a possible solution lies in the exploitation of the environmental kinetic energy for generating electrical energy through piezoelectric vibration energy harvesting systems [1], [2]. These functional structures, at the MEMS scale, are characterized by high natural frequency of vibration in contrast to the typical frequencies of the environment that are very small. Due to this mismatch, the vibration of the harvester is in practice not activated and very low levels of electrical energy can be obtained. In order to solve this problem, an efficient technique to upconvert the frequency of the input signal through magnetic interaction is here presented as also proposed in [3]. A theoretical and computational study is performed on a high-frequency layered piezoelectric cantilever with a silicon substrate and a PZT functional layer. This choice of laminate is fully compatible with the current micromachining process in which the deposition of the active layer is made by sol-gel or sputtering techniques. Dynamic analyses are performed under different input conditions of motion and the frequency up-conversion (FuC) occurs due to the interaction of the cantilever with a low frequency mass through Neodymium permanent magnets. Beyond the FuC mechanism, also an experimental investigation of the magnetic interaction is presented, pointing out some critical issues of typical analytical approaches with respect to real physics in terms of energy. This reflects on the performance of the functional structure.
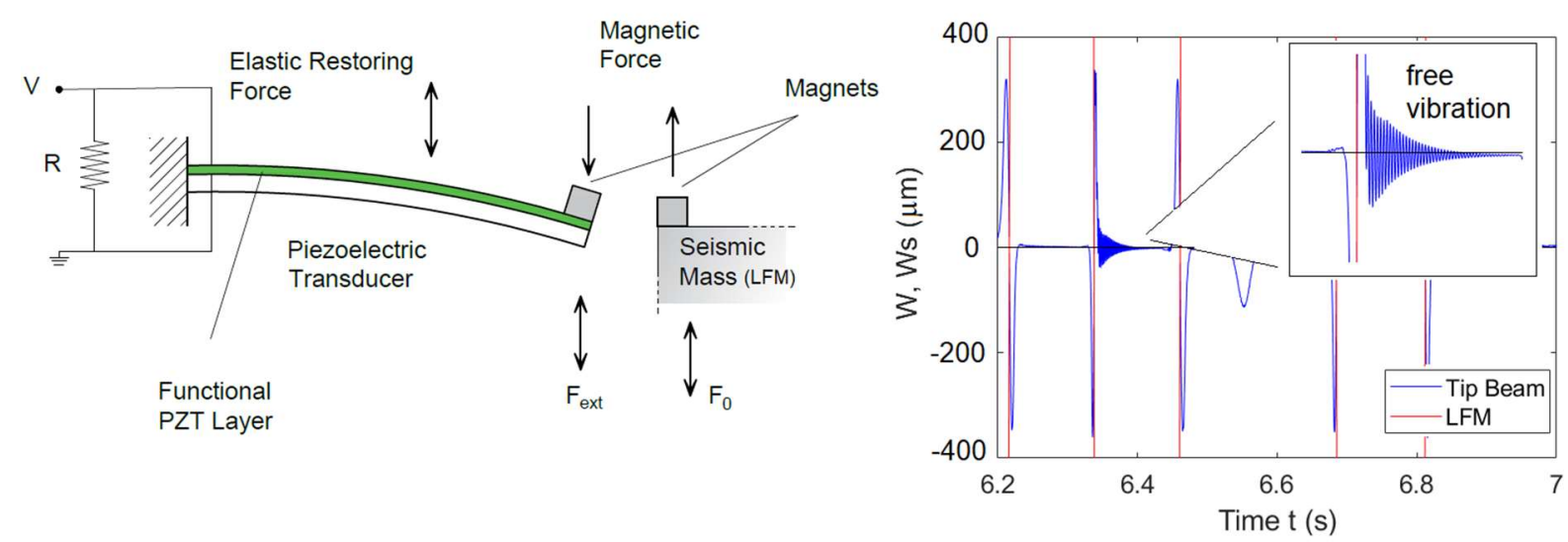

Figure 1. Concept of the up-conversion technique for a functional laminate cantilever

\section{References}

[1] A.Erturk, D.J. Inman, Piezoelectric Energy Harvesting, 2011, Wiley

[2] A. Corigliano, R. Ardito, C. Comi, A. Frangi, A. Ghisi, S. Mariani, Mechanics of Microsystems, 2018, Wiley

[3] Q.C. Tang, Y.L. Yang, X. Li, Bi-stable frequency up-conversion piezoelectric energy harvester driven by non-contact magnetic repulsion, Smart Materials and Structures, 2011, 20,12501 